\title{
Observations and numerical simulations of gradual SEP events with Ulysses and ACE
}

\author{
L.-L. Lian, ${ }^{a, *}$ G. Qin, ${ }^{a}$ Y. Wang ${ }^{a}$ and S.-W. Cui ${ }^{b}$ \\ ${ }^{a}$ School of Science, Harbin Institute of Technology, Shenzhen 518055, China \\ ${ }^{b}$ Hebei Normal University, Shijiazhuang, 050024, China \\ E-mail: 1llian@hebtu.edu.cn, qingang@hit.edu.cn
}

\begin{abstract}
We study the latitudinal extent of the gradual solar energetic particle (SEP) events of 2000 June 10 and 2001 December 26 using energetic electron observations from the ACE and Ulysses. We investigate the effects of particle source and transport on the profiles. We get the best fit parameters for simulations by comparing simulations with the two spacecraft observations. We show that perpendicular diffusion can significantly affect the propagation of particles. In addition, it is found that the start and peak times of particle injections are between the onset and peak times of flare for the two events. Furthermore, we have models for the peak intensity of the particle source and the time interval from the onset of flares to the peak time of the particle source. We show that the models agree well with the best fit parameters.
\end{abstract}

$37^{\text {th }}$ International Cosmic Ray Conference (ICRC 2021)

July 12th - 23rd, 2021

Online - Berlin, Germany

\footnotetext{
*Presenter
} 


\section{Introduction}

In order to study particles acceleration and transport in the heliosphere, it is necessary to use simultaneous observations of solar energetic particle (SEP) time profiles from multiple spacecraft, which show great diversity in different interplanetary space locations [e.g., 1-4].

Previously, most of the numerical works of SEPs and their comparison with observations are done with spacecraft separated in longitudinal and radial extents [e.g., 5-7]. However, there are still some simulations and analyses with spacecraft observations at different latitudes [e.g., 8]. In this paper, to focus on the latitudinal extent of SEPs transport in interplanetary space, we study two gradual SEP events with electrons of energies $>53 \mathrm{keV}$ observed by both the near-Earth spacecraft, $A C E$, and the high-latitude one, Ulysses. We discuss the effects of transport processes and injection source on the SEP intensities by comparing our simulation results with the multiple spacecraft observations.

\section{Observations}

In this work, we select two gradual SEP events of 2000 June 10 and 2001 December 26, which were both observed by the ACE/EPAM [9] and Ulysses/HISCALE [10] located at different longitudes, latitudes, and radial distances.

For the gradual SEP event of 2000 June 10, the solar origin of the SEP event has been associated with an intense M5.2 flare which started to increase at 16:40 UT and peaked at 17:02 UT in AR9026 at N22W38 (https: / / www . spaceweatherlive . com) and a 1008 km/s halo CME. For the event, the $A C E$ was in the ecliptic at $1 \mathrm{au}$, and Ulysses was about 3.37 au, latitude being S58, and longitude being $87^{\circ}$ to the east with respect to the Earth. The solar wind speeds observed by $A C E$ and Ulysses were $V_{A C E}^{\mathrm{SW}}=505 \mathrm{~km} \mathrm{~s}^{-1}$ and $V_{\text {Ulysses }}^{\mathrm{SW}}=475 \mathrm{~km} \mathrm{~s}^{-1}$, respectively.
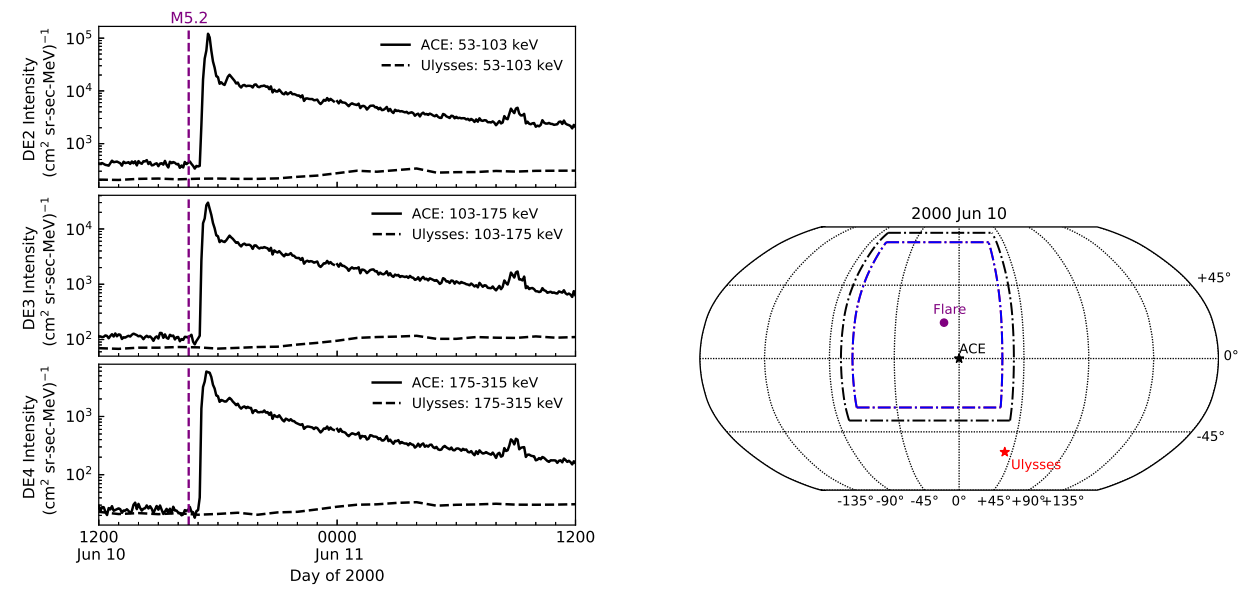

Figure 1: The left panels show the observation results of ACE (solid lines) and Ulysses (dashed lines) in DE2DE4 energy channels, and the right panels shows the footpoints of ACE and Ulysses, as well as the flare, on the solar surface.

In the left panels of Figure 1, from top to bottom, electron intensities measured in three different energy channels of ACE and Ulysses are plotted with solid and dashed lines, respectively. 
The vertical line indicates the start time of flare with $\sim 8$ minute light travel time from Sun to 1 au reduced. In the left panels shows on the solar surface the footpoints of ACE and Ulysses, as well as the flare, using the footpoint of ACE as the coordinate origin. Although the Ulysses footpoint is far away from the center of the particle source, Figure 1 clearly shows that both spacecraft detected particle intensity enhancements in the DE2-DE4 energy channels. For these energy channels, the particle intensities observed by $A C E$ increased dramatically shortly after the flare eruption, while the intensities on Ulysses increased slowly. Besides, the electron peak intensity of ACE occurred earlier than that of Ulysses. Moreover, the $A C E$ peak intensities are about 2 order of magnitude of the Ulysses in DE2-DE4 energy channels. Meanwhile, we can see that ACE flux had a faster decay than Ulysses during the decay phase. It is suggested that the difference between the time-intensity profiles of the two spacecraft can be attributed to the different locations of $A C E$ and Ulysses with respect to the particle source.

The gradual SEP event of 2001 December 26 was also observed by both $A C E$ and Ulysses spacecraft, with the solar origin associated with an intense M7.1 flare beginning at 04:32 UT and peaking at 05:40 UT from AR9742 at N08W54, and a CME with plane-of-sky speeds above 1446 $\mathrm{km} / \mathrm{s}$ and angular width larger than $212^{\circ}$. In addition, Ulysses was about 2.54 au, latitude being N69, and longitude being $39^{\circ}$ to the west with respect to the Earth. The solar wind speeds observed by $A C E$ and Ulysses were $V_{A C E}^{\mathrm{SW}}=380 \mathrm{~km} \mathrm{~s}^{-1}$ and $V_{\text {Ulysses }}^{\mathrm{SW}}=575 \mathrm{~km} \mathrm{~s}^{-1}$, respectively.
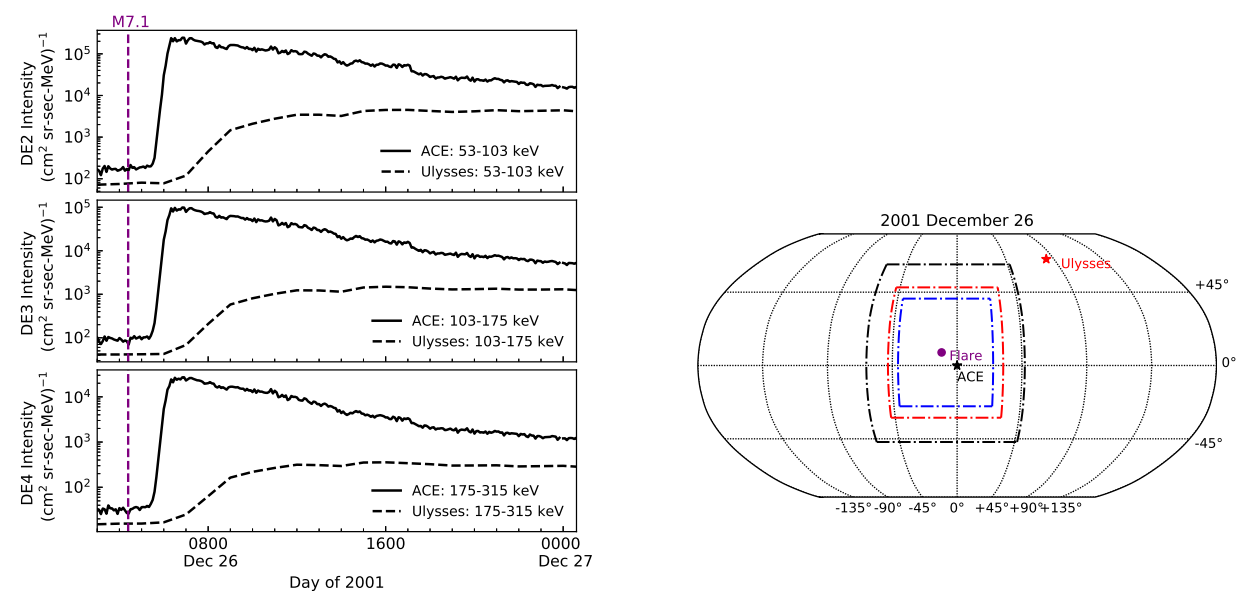

Figure 2: Same as Figure 1, except that it is for the event of 2001 December 26.

Figure 2 shows, using the same format as Figure 1, the time series of SEP fluxes and the footpoint of each spacecraft. For this event, both spacecraft detected significant particle intensity enhancements despite a large separation in longitude at Ulysses. It is also seen from Figure 2 that electron intensity started to increase immediately after the solar flare at $A C E$ (solid lines), but energetic particles did not increase immediately at Ulysses (dashed lines) until about one hour later and the increase was gradual compared to ACE observation. The peak intensity at Ulysses was much smaller and the occurrence of maximum intensity was several hours later than $A C E$. 


\section{Models}

We have modeled SEP interplanetary transport to ACE and Ulysses during the two selected SEP events by numerically solving the three-dimensional focused transport equation ([11]). The forcused transport equation of SEPs can be written as [11-13]

$$
\begin{aligned}
& \frac{\partial f}{\partial t}=\nabla \cdot\left(\overrightarrow{\kappa_{\perp}} \cdot \nabla f\right)+\frac{\partial}{\partial \mu}\left(D_{\mu \mu} \frac{\partial f}{\partial \mu}\right)-\left(v \mu \overrightarrow{\hat{b}}+\vec{V}^{\mathrm{SW}}\right) \cdot \nabla f \\
& +p\left[\frac{1-\mu^{2}}{2}\left(\nabla \cdot \vec{V}^{\mathrm{SW}}-\hat{\hat{b}} \hat{b}: \nabla \vec{V}^{\mathrm{SW}}\right)+\mu^{2} \hat{b} \hat{b}: \nabla \vec{V}^{\mathrm{SW}}\right] \frac{\partial f}{\partial p} \\
& -\frac{1-\mu^{2}}{2}\left[-\frac{v}{L}+\mu\left(\nabla \cdot \vec{V}^{\mathrm{SW}}-3 \hat{b} \hat{b}: \nabla \vec{V}^{\mathrm{SW}}\right)\right] \frac{\partial f}{\partial \mu},
\end{aligned}
$$

where $f(\vec{x}, \mu, p, t)$ is gyrophase-averaged distribution function, $\overrightarrow{\kappa_{\perp}}$ is the perpendicular diffusion $\vec{\wedge}$ coefficient, $D_{\mu \mu}$ is pitch angle diffusion coefficient, $b$ is a unit vector along the local magnetic field, $\vec{V}^{\mathrm{SW}}$ is the solar wind velocity in the radial direction, and $L$ is the magnetic focusing length, with $B_{0}$ being the magnitude of the background IMF set as the Parker field.

The source particles are injected at $r \leq 0.05 \mathrm{au}$. Its boundary condition is chosen to have the following form:

$$
Q\left(z \leq 0.05 \mathrm{au}, E_{\mathrm{k}}, \theta, \varphi, t\right)=\frac{C}{t} \frac{E_{\mathrm{k}}^{-\gamma}}{p^{2}} \exp \left(-\frac{\tau_{c}}{t}-\frac{t}{\tau_{\mathrm{L}}}\right),
$$

where $E_{k}$ is the particle's kinetic energy, $\gamma=3$ is the spectral index of source particles, $\tau_{c}$ and $\tau_{L}$ (in units of days) are the rise and decay timescales of source injection profile, respectively.

Zhang [15] introduced the time-backward Markov stochastic process method to solve cosmic ray transport equation, which was adopted by Qin et al. [13] to get the solution of SEPs transport equation and compare with spacecraft observations. The details of this method can be found in Zhang [15] and Qin et al. [13].

\section{The SEP simulation results and comparison with observations}

For the 2000 June 10 SEP event, to fit the simulations with the observations, we get the best fit parameters shown in Table 1. In left panel of Figure 3 purple line shows the flare intensity observed by $G O E S$, and vertical lines show the onset and peak times of flare. The time interval between the two vertical lines, i.e., the time from initial to peak time of the X-ray flare, $\Delta T_{\mathrm{d}}$, is 22 min. From this panel we can see that the particle source onset and peak times were between the onset and the peak times of the flare in all three energy channels. In the right panels of Figure 3 shows the electron time-intensity profiles from observations and simulations. We use the geometrical mean energy as the simulated energies, i.e., $74 \mathrm{keV}, 134 \mathrm{keV}$, and $235 \mathrm{keV}$, for energy channels DE2, DE3, and DE4, respectively. It can be seen from Figure 3 that the simulations successfully reproduce most features of the observations in the energy channels of DE2-DE4.

Figure 4 is similar as Figure 3 except that it is for the SEP event of 2001 December 26. For this event, to fit the simulations with the observations, we also get the best fit parameters shown in 

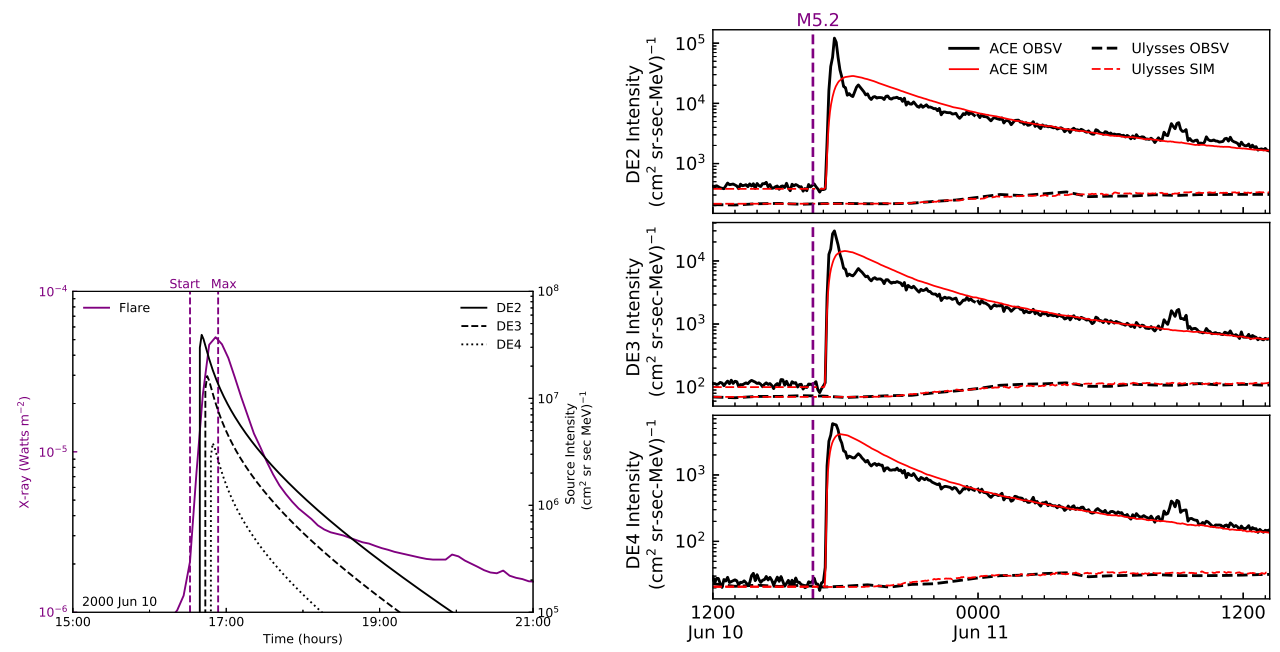

Figure 3: The left panels show the particle source profiles in DE2 DE4 and associated SXR flare, and the right panels shows the comparison of the observations of electron intensities and the simulation results for the event of 2000 June 10.
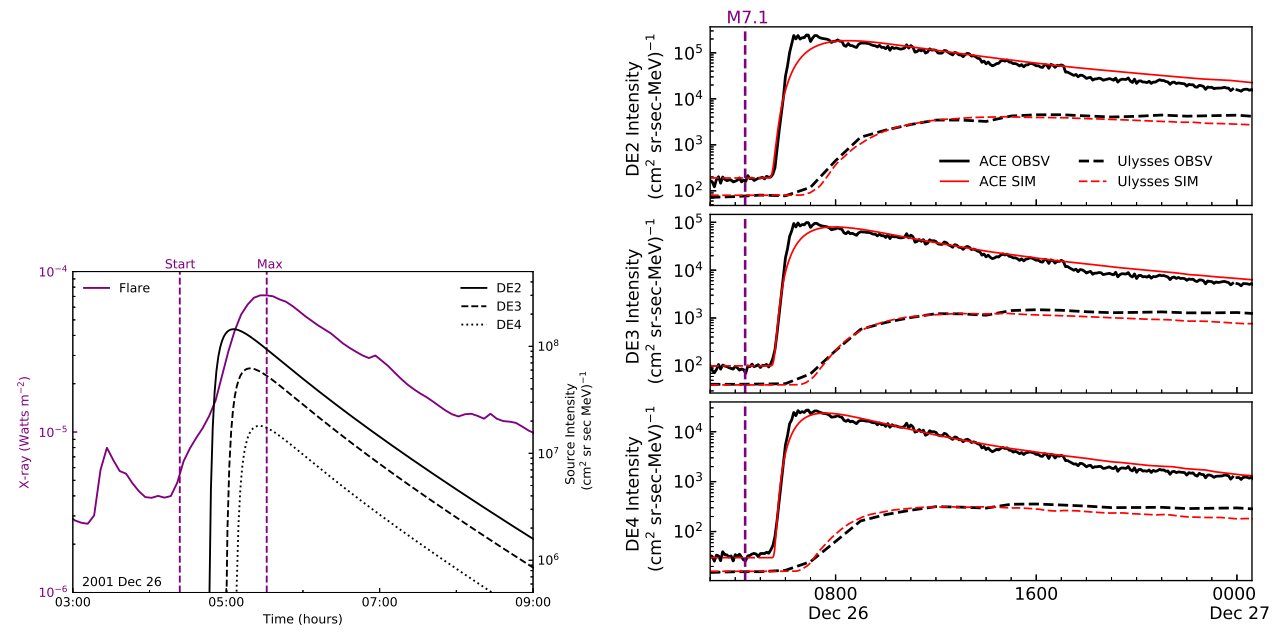

Figure 4: Same as Figure 3, except that it is for the event of 2001 December 26.

Table 1. From the best fit parameters and the particle source model Equation (2) we also have the time-intensity profiles of particle sources in different energy channels in left panel of Figure 4. It is shown that $\Delta T_{\mathrm{d}}$ equals to $68 \mathrm{~min}$. It is also shown that the particle source onset and peak times were between the onset and the peak times of the flare in DE2-DE4. In addition, the onset and peak times of the particle source for higher energy particles are later than that for lower energy particles. In right panels of Figure 4 is similar as Figure 3 to show the electron time-intensity profiles from observations and simulations for the energy channels of DE2-DE4. It can be seen from Figure 4 that, the simulations reproduce most features of the observations in the energy channels of DE2-DE4. 
Table 1: Best fit parameters for the simulations.

\begin{tabular}{ccccccc}
\hline \hline Event Date & Channel & $\begin{array}{c}\tau_{\mathrm{c}} \\
(\text { day })\end{array}$ & $\begin{array}{c}\tau_{\mathrm{L}} \\
(\text { day })\end{array}$ & $\begin{array}{c}\Delta \theta \\
\left({ }^{\circ}\right)\end{array}$ & $\begin{array}{c}I_{\max } \\
\left(\mathrm{cm}^{2} \mathrm{sr} \mathrm{s} \mathrm{MeV}\right)^{-1}\end{array}$ & $\begin{array}{c}\Delta T_{1} \\
(\mathrm{~min})\end{array}$ \\
\hline \multirow{3}{*}{ 2000 Jun 10 } & DE2 & 0.002 & 0.05 & 60 & $3.92 \mathrm{E}+07$ & 6.32 \\
& DE3 & 0.002 & 0.05 & 53 & $1.61 \mathrm{E}+07$ & 10.64 \\
& DE4 & 0.002 & 0.05 & 53 & $3.47 \mathrm{E}+06$ & 14.96 \\
\hline \multirow{3}{*}{ 2001 Dec 26 } & DE2 & 0.02 & 0.05 & 55 & $1.45 \mathrm{E}+08$ & 20.08 \\
& DE3 & 0.02 & 0.05 & 40 & $6.22 \mathrm{E}+07$ & 33.04 \\
& DE4 & 0.02 & 0.05 & 33 & $1.81 \mathrm{E}+07$ & 40.24 \\
\hline
\end{tabular}

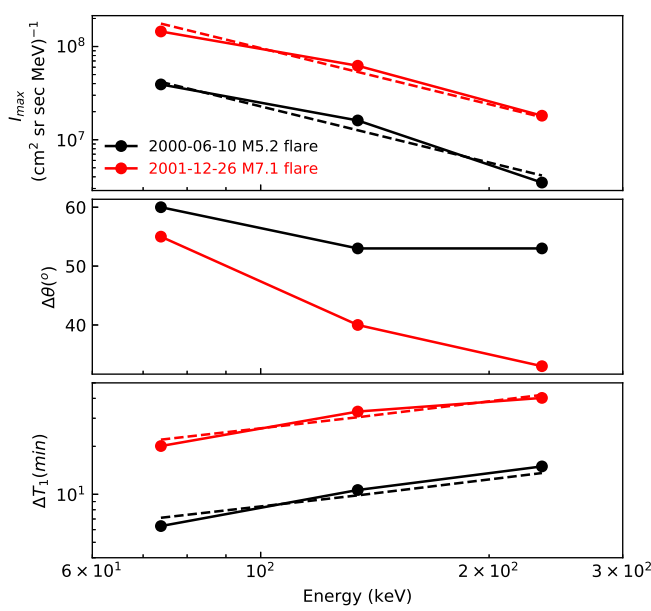

Figure 5: From top to bottom: the maximum value of particle source intensity $I_{\max }$, the half width of particle source $\Delta \theta$, and the time interval from the onset time of the flare to the peak time of the particle source, $\Delta T_{1}$, with different energy for the two SEP events. Solid circles connected by solid lines indicate the best fit parameters, and the dashed lines indicate the modeling results.

\section{Best fit parameters for particle source and the models}

We study the best fit parameters for the particle source in Table 1 in the energy channels DE2-DE4. It is shown that the particle source parameters $\tau_{c}$ and $\tau_{L}$ are the same in the same event, but the peak values of the particle source intensity $I_{\max }$, the half width of particle source $\Delta \theta$, and the time interval from the onset time of the flare to the peak time of the particle source $\Delta T_{1}$ vary with energy.

Figure 5 shows best fit parameters $I_{\max }, \Delta \theta$, and $\Delta T_{1}$ as function of energy as solid circles in top, middle, and bottom panels, respectively, for different events, where black and red color indicate the events 2000 June 10 and 2001 December 26, respectively.

The top panel of Figure 5 shows that for both of the events $I_{\max }$ decreases as the increasing of energy $E$. In addition, for the same energy channel, $I_{\max }$ is higher in the 2001 December 26 event which has larger SXR peak intensity, $F_{\text {SXR }}$, and the time interval from the SXR initial time to the peak time, $\Delta T_{\mathrm{d}}$. One may assume that the total energy released by a flare is proportional to the product of flare peak intensity and the rise time, i.e., $F_{\mathrm{SXR}} \Delta T_{\mathrm{d}}$. On the other hand, the number of energetic electrons of the SEP released is assumed proportional to $I_{\max } E$, so the total energy of 
energetic electrons of the SEP released may be proportional to $I_{\max } E^{2}$. Although it is assumed that the energetic electrons in a gradual SEP event are accelerated by the coronal shock, the strength of the flare can be used to indicate that of the solar event. Therefore, one may assume that the total energy released by a flare is proportional to the energy released by the energetic electrons,

$$
I_{\max }=\frac{G F_{\mathrm{SXR}} \Delta T_{\mathrm{d}}}{E^{2}},
$$

where $G$ is a constant to be determined. In top panel of Figure 5 the dashed lines show the model results from Equation (3) with the best fit value $G=2.07 \times 10^{-3}(\mathrm{~s} \mathrm{sr})^{-1}$. It is shown that the modeling results agree well with the best fit parameters.

The middle panel of Figure 5 shows that for both events the best fit parameter $\Delta \theta$ decreases as the increasing of energy $E$. The reason might be that it is more difficult to accelerate electrons to higher energies. Furthermore, $\Delta \theta$ is smaller in event 2001 December 26 than that in event 2000 June 10.

The bottom panel of Figure 5 shows that for both events the best fit parameter $\Delta T_{1}$ increases as the increasing of energy $E$. It is also shown that $\Delta T_{1}$ is larger in the 2001 December 26 event with stronger flare. It is assumed that to accelerate the particles to higher momentum, longer time is needed, and that an event with longer rise time of flare $\Delta T_{\mathrm{d}}$ can accelerate particles longer, or to make $\Delta T_{1}$ larger, so that we may suggest

$$
\Delta T_{1}=H p \Delta T_{\mathrm{d}},
$$

where $H$ is a constant to be determined. In the bottom panel of Figure 5 dashed lines show the modeling results from Equation (4) with the best fit value $H=3.41 \times 10^{11}(\mathrm{GeV} / \mathrm{c})^{-1}$. It is shown that the modeling results generally agree with the best fit parameters.

\section{Summary and discussion}

In this work, we study two SEP events of 2000 June 10 and 2001 December 26 which were supposed to each originate from an intense coronal shock with a solar flare, to focus on the latitudinal extent of SEPs. The SEPs were observed by both ACE in ecliptic and Ulysses in high latitude. We do data analysis of the observational results from energy channels DE2, DE3, and DE4 of the spacecraft. In addition, we do simulations by solving the three-dimensional focused transport equation of SEPs, which includes many important transport mechanisms, such as particle streaming along the field line, adiabatic cooling, magnetic focusing, and the diffusion perpendicular and parallel to the background magnetic field.

With different energies we do simulations for the two SEP events, to obtain the SEPs timeintensity profiles for different spacecraft. We try different fitting parameters to get simulation results for the SEP events to compare with ACE and Ulysses observations, so we can get the best fit parameters.

For the best fit parameters of the energetic particle source with the SEP events of 2000 June 10 and 2001 December 26, all the onset times and peak times of the particle source are between the start and peak times of the flare. Moreover, it is found that the peak values of the particle source intensity $I_{\max }$ decreases as the increasing of energy, and increases with stronger solar flare. 
Accordingly, we have a model for the peak value of the source intensity $I_{\max }$ proportional to $F_{\mathrm{SXR}}$, $\Delta T_{\mathrm{d}}$, and $E^{-2}$, which agrees well with the best fit parameters. In addition, we find that the best fit half width of particle source $\Delta \theta$ decreases with the increasing of energy, the reason might be the difficulty to accelerate electrons to higher energies. Furthermore, it is shown that the best fit time interval from the onset time of the flare to the peak time of the particle source $\Delta T_{1}$ increases as the increasing of energy. We have a model for $\Delta T_{1}$ proportional to momentum $p$ and $\Delta T_{\mathrm{d}}$, which generally agree with the fitting results from simulations.

\section{Acknowledgements}

This work was supported by grants NNSFC 42074206, NNSFC 41874206, and U1831129.

\section{References}

[1] Richardson, I. G., von Rosenvinge, T. T., Cane, H. V., et al. 2014, Solar Physics, 289, 3059

[2] Desai, M., \& Giacalone, J. 2016, LRSP, 13, 3

[3] Paassilta, M., Papaioannou, A., Dresing, N., et al. 2018, Solar Physics, 293, 70

[4] Wang, Y., Lyu, D., Xiao, B. X., et al. 2021, The Astrophysical Journal, in press

[5] Qin, G., \& Wang, Y. 2015, The Astrophysical Journal, 809, 177

[6] Dröge, W., Kartavykh, Y. Y., Dresing, N., \& Klassen, A. 2016, The Astrophysical Journal, 826,134

[7] Zhao, L. L., \& Zhang, M. 2018, The Astrophysical Journal, 859, L29

[8] Agueda, N., Vainio, R., Dalla, S., Lario, D. \& Sanahuja, B. 2013, The Astrophysical Journal 765,83

[9] Gold, R. E., Krimigis, S. M., Hawkins, S. E. III, et al. 1998, Space Science Reviews, 86, 541

[10] Lanzerotti, L. J., Gold, R. E., Anderson, K. A., et al. 1992, Astronomy and Astrophysics Supplement Series, 92, 349

[11] Zhang, M., Qin, G., \& Rassoul, H. 2009, The Astrophysical Journal, 692, 109

[12] Skilling, J. 1971, The Astrophysical Journal, 170, 265

[13] Qin, G., Zhang, M., \& Dwyer, J. R. 2006, Journal of Geophysical Research, 111, A08101

[14] Reid, G. C. 1964, Journal of Geophysical Research, 69, 2659

[15] Zhang, M. 1999, The Astrophysical Journal, 513, 409 